\title{
Three-way electrical gating characteristics of metallic Y-junction carbon nanotubes
}

\author{
J. Park, C. Daraio, S. Jin, and P. R. Bandaru ${ }^{\text {a) }}$ \\ Materials Science and Engineering Program, Mechanical and Aerospace Engineering, \\ University of California, San Diego, La Jolla, California 92093-0411 \\ J. Gaillard and A. M. Rao \\ Department of Physics and Astronomy, Clemson University, Clemson, South Carolina 29634
}

(Received 3 February 2006; accepted 18 May 2006; published online 15 June 2006)

\begin{abstract}
Y-junction based carbon nanotube (CNT) transistors exhibit interesting switching behaviors, and have the structural advantage that the electrical gate for current modulation can be formed by any of the three constituent branches. In this letter, we report on the gating characteristics of metallic Y-CNT morphologies. By measuring the output conductance and transconductance we conclude that the efficiency and gain depend on the branch diameter and is electric field controlled. Based on these principles, we propose a design for a Y-junction based CNT switching device, with tunable electrical properties. () 2006 American Institute of Physics. [DOI: 10.1063/1.2213013]
\end{abstract}

While conventional silicon microfabrication technology encounters barriers to further development due to difficulties in miniaturization and increased cost, carbon nanotubes (CNTs) show promise for a molecular electronics ${ }^{1,2}$ based technology. CNTs [both single walled nanotubes (SWNTs) and multiwalled nanotubes (MWNTs)] can be synthesized to be either semiconducting or metallic. ${ }^{3}$ Consequently, they can be used in a wide variety of electronics including diodes, transistors, and high frequency devices. ${ }^{4}$ The ultimate aim is an integrated nanoelectronics system, with the advantages of reduced power consumption, radiation hardness, and faster speeds of operation, which incorporates CNTs as both active devices and interconnects. The electrical and thermal conductivity $^{5}$ properties of both SWNTs (Ref. 6) and MWNTs (Ref. 7) have been well explored. While clean SWNTs (diameter $\sim 1 \mathrm{~nm}$ ) can be described as quantum wires due to the ballistic nature of electron transport, ${ }^{8}$ the transport in MWNTs (diameter in the range of $2-100 \mathrm{~nm}$ ) is found to be diffusive/quasiballistic. ${ }^{7}$ On this basis, Coulomb blockade $^{9}$ and field effect ${ }^{10}$ phenomena have been used for demonstrating nanotube based transistors. While extremely important in elucidating fundamental properties, the above experiments have used external electrodes made through conventional lithographic processes to contact the nanotubes and do not truly represent nanoelectronic circuits. Additionally, the well known metal oxide semiconductor field effect transistor (MOSFET) architecture is used, where the nanotube serves as the channel between the electrodes (source and drain), and $\mathrm{SiO}_{2} / \mathrm{Si}$ based gate modulates the channel conductance. In other demonstrations of electrical switching, cumbersome atomic force microscope (AFM) manipulations ${ }^{11}$ of nanotube properties have been utilized.

It would, therefore, be very attractive to propose different types of electronic elements and paradigms of switching, to harness functionalities peculiar to CNT forms ${ }^{12}$ such as $Y$ and $T$ junctions. ${ }^{13}$ In addition to the nanometer size of these devices, there is also the additional feature of having a selfcontained gate, i.e., in a switching device, the gate does not

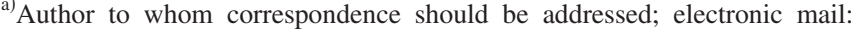
pbandaru@ucsd.edu
}

have to be separately fabricated but forms a part of the structure. In earlier experiments, rectification, ${ }^{14}$ switching, and logic gates ${ }^{15}$ were demonstrated in Y-shaped CNTs. An abrupt switching action through two branches of the $\mathrm{Y}$ junction, when a control/gating dc voltage was applied to the third terminal was seen. It was also proposed that all three branches of a Y-CNT could be possibly used for a gate, which we expand upon in this letter. We report on the current $(I)$-voltage $(V)$ characteristics of a metallic Y-CNT and relate the gating efficiency of each branch, together with the voltage and current gain, to the nanostructure geometry.

The Y-CNTs were synthesized by a modified chemical vapor deposition (CVD) technique. ${ }^{16}$ Subsequent to synthesis, the Y-CNTs were collected from the CVD chamber and individually separated by ultrasonication in an ethanol solvent. They were then dispersed on a Au electrode patterned $\mathrm{SiO}_{2} / \mathrm{Si}$ substrate. As observed through transmission electron microscopy, ${ }^{7}$ the Y-CNTs comprise multiwalled nanotubes of the bamboo ${ }^{17}$ variety, and a detailed examination of the nanostructure reveals the presence of randomly dispersed catalyst particles. A scanning electron microscope (SEM) was used to locate the nanotubes and focused ion beam (FIB) induced deposition was used to lay down Pt lines to the three Y terminals. Any possible ion-induced damage to the CNTs was avoided through low operating voltages. It was verified that this procedure results in high-quality Ohmic contacts, with contact resistances of the order of $1 \mathrm{k} \Omega{ }^{18}$

The experimental setup for the electrical characterization is implemented on a Wentworth probe station and is shown in Fig. 1. A dc control voltage $(V)$ is applied on one of the three terminals (gate) of the Y-CNT while the current $(I)$ through the other two terminals (source and drain) is monitored. Phase sensitive electrical measurements using a lock-in amplifier were also employed and qualitatively similar results were obtained on many samples. The $I-V$ curves (Fig. 1) of the Y-CNTs, as a function of gate $\left(V_{\mathrm{gs}}\right) /$ bias $\left(V_{\mathrm{ds}}\right)$ voltage, are shown in Fig. 2. The notable features are (a) Ohmic conduction, (b) a proportional displacement of the current with $V_{\mathrm{gs}}$ and $V_{\mathrm{ds}}$, and (c) a geometry dependent conductance. The devices are also seen to have both current and voltage gains. In line with an earlier ${ }^{19}$ study, we define the 


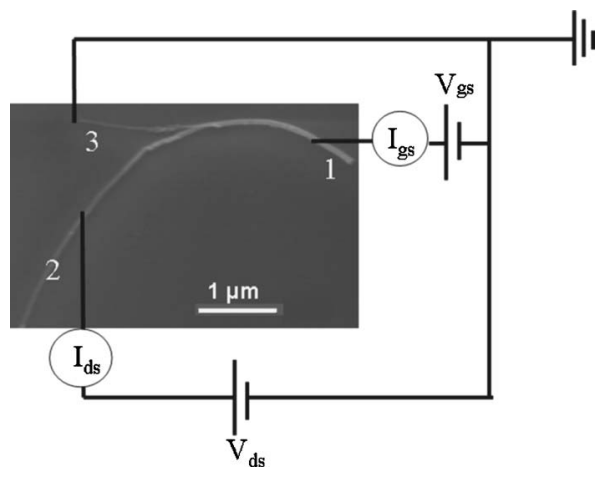

FIG. 1. (Color online) The circuit arrangement used to probe the three-way gating capability of a Y-CNT switching device (SEM micrograph). The Y-CNT is contacted by FIB deposited Pt lines to Ti/Au bonding pads (not shown). In the diagram, 1 is the control gate (g) while 2 and 3 are the source (s) and drain (d), respectively. A complete evaluation of electrical characteristics was implemented by implementing each terminal $(1,2$, and 3$)$ successively as the gate while probing the electrical conduction through the other two branches.

differential current gain $\left(\mathbf{g}_{\text {diff }}\right)$ defined as the ratio of $\left(\partial I_{\mathrm{gs}} / \partial V_{\mathrm{gs}}\right)$ to $\left(\partial I_{\mathrm{ds}} / \partial V_{\mathrm{gs}}\right)$, at a constant source-drain voltage $\left(V_{\mathrm{ds}}\right)$. In our Y-CNT devices a $\mathbf{g}_{\mathrm{diff}}$ of up to 10 is obtained at room temperature. The voltage gain is calculated ${ }^{10}$ by considering the voltage change $\left(\Delta V_{\mathrm{ds}}\right)$ for a given increment in gate voltage $\left(\Delta V_{g}\right)$, at a constant value of the current. The voltage gain when the stem (1 in Fig. 1) is used for electrical gating is $\sim 6$ [Fig. 2(b)], while the value drops to $\sim 0.3$ [Fig. 2(d)] when the branch (3 in Fig. 1) is used for a gate.

Other quantities of importance in quantifying the electrical characteristics are the transconductance, $\mathbf{g}_{m}$ $=\left(\partial I_{\mathrm{ds}} / \partial V_{\mathrm{gs}}\right)_{V_{\mathrm{ds}}}$, the ratio of the output current $\left(I_{\mathrm{ds}}\right)$ to the modulating/gating voltage $\left(V_{\mathrm{gs}}\right)$ at constant bias voltage $\left(V_{\mathrm{ds}}\right)$, and the output conductance, $\mathbf{g}_{d}=\left(\partial I_{\mathrm{ds}} / \partial V_{\mathrm{ds}}\right)_{V_{\mathrm{gs}}}$, the ratio of the output current to the bias voltage at a constant gate voltage. The results for all the three possible configurations of gate (g), with the other two terminals as source (s) and drain (d) are shown in Fig. 3(a), and summarized in the inset.
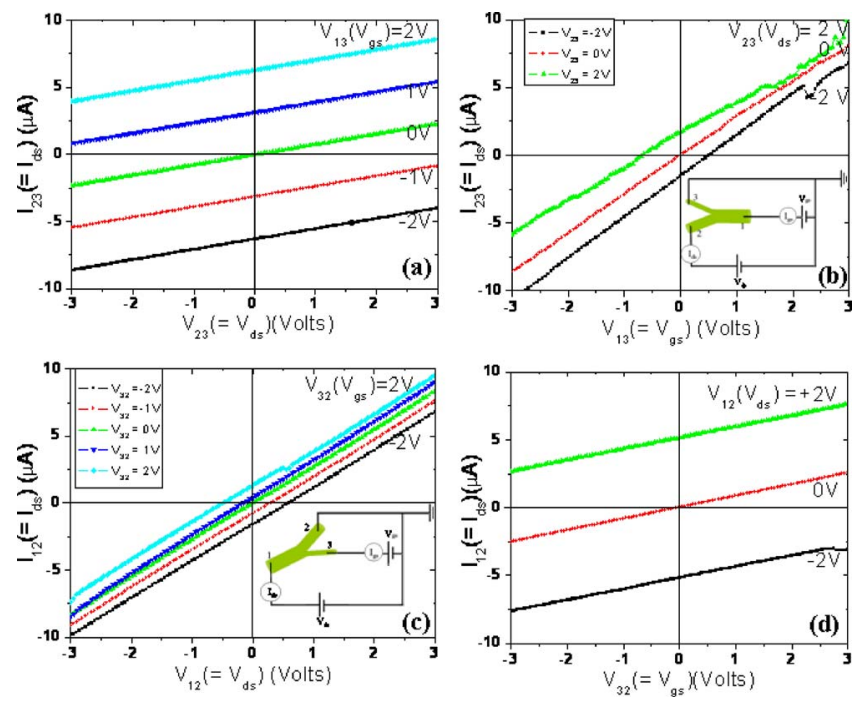

FIG. 2. (Color online) Current $(I)$-Voltage $(V)$ characteristics for the Y-CNT. (a) $I_{23^{-}}-V_{23}\left(=V_{\mathrm{ds}}\right)$ with terminal 1 as the gate $\left(V_{\mathrm{gs}}=V_{13}\right)$. (b) $I_{23^{-}} V_{13}\left(=V_{\mathrm{gs}}\right)$ as a function of the bias voltage $V_{23},\left(=V_{\mathrm{ds}}\right)$ applied across branches 2 and 3 , and (c) $I_{12}-V_{12}$ with terminal 3 as the gate $\left(V_{13}=V_{\mathrm{gs}}\right)$. (d) $I_{23}-V_{32}\left(=V_{\mathrm{ds}}\right)$ as a function of the bias voltage $V_{12}\left(=V_{\mathrm{ds}}\right)$ applied across stem 1 and branch 2 .
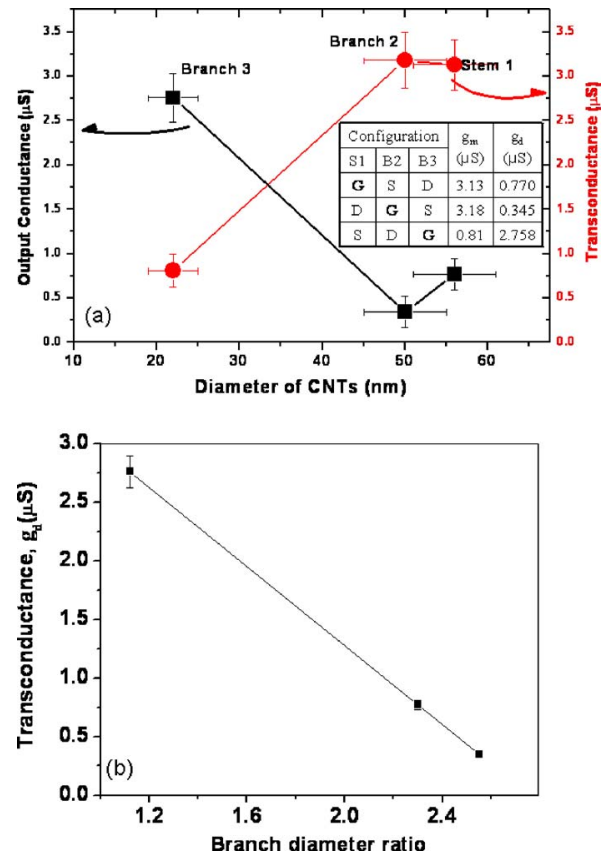

FIG. 3. (Color online) (a) The variation of the output conductance $\left(\mathrm{g}_{d}\right)$ and transconductance $\left(\mathrm{g}_{m}\right)$ with the diameters of the constituent branches of the Y-CNT. The inset table summarizes the data for all the possible permutations of the gate terminal. (b) The conductance $\left(\mathrm{g}_{d}\right)$ through two nanotubes of different diameters in the Y-CNT is inversely proportional to their ratio $\left(=d_{>} / d_{<}\right)$.

The values have been normalized to equivalent lengths of the constituent nanotubes. It is found that the magnitude of the $\mathbf{g}_{d}$ is inversely proportional to the ratio of the diameters of the branches through which the current is flowing [Fig. 3(b)], i.e., a larger discrepancy between the branch diameters lowers the conductance. On the other hand, the $\mathbf{g}_{m}$ which denotes the gating efficiency for current modulation, seems to be directly proportional to the diameter of the gating nanotube.

While each of the constituent nanotubes in the Y-CNT is metallic, the necessity of maintaining a uniform electrochemical potential $(\mu=-\mathrm{eV})$ in the overall nanostructure gives rise to nonuniform/nonlinear interactions. ${ }^{20}$ For example, when $V_{2}$ (the voltage on branch 2) decreases, $\mu_{2}\left(=-\mathrm{eV}_{2}\right)$ increases, and an excess electron current flows towards the central junction. The balance between the incoming current and the outgoing currents, at the junction itself, is achieved by increasing $\mu_{1}$ (decreasing $V_{1}$ ). On the other hand, when $\mu_{2}$ decreases, $\mu_{1}$ decreases also, but cannot decrease past a certain critical point, viz., the fixed electrochemical potential, dictated by geometry/defects, of the junction. This limitation results in a saturation of the current, and is observed in Fig. 2(b).

Due to the interconnected nature of the $\mathrm{Y}$ junction, the gating is not perfect and there is a current leakage which is manifested in the $I-V$ curves, i.e., the current is diverted from one path (say, 2-3) to another (say, 1-2) when a gating voltage is applied on 1, and results in current displacement. However, at a certain fixed voltage $\left(V_{\mathrm{ds}}\right)$, the control voltage $\left(V_{g}\right)$ can be modulated and it is seen that positive, zero, or negative currents can flow through the device, which could be interpreted as switching. While operation up to $100 \mathrm{kHz}$ is possible, the upper limit seems to be set by the nanotube capacitance related to the details of the Y-CNT structure. This switching is of a different form than the one observed in o AlP license or copyright, see http://apl.aip.org/apl/copyright.jsp 
(a)

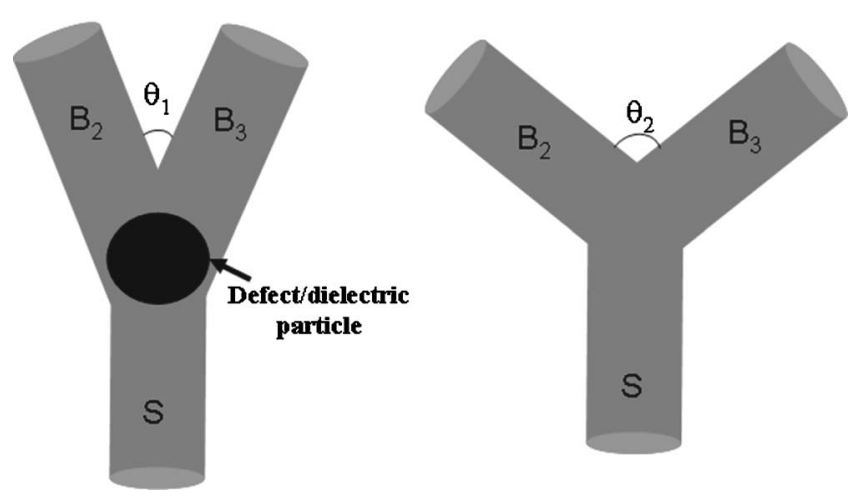

FIG. 4. (Color online) (a) A proposed Y-junction CNT based switching device, where a smaller angle $\left(\theta_{1}\right)$ between the branches $\left(B_{2}\right.$ and $\left.B_{3}\right)$ can result in a higher gating efficiency for the stem (S). A defect at the junction region can also help (see text). (b) A Y-CNT with uniform gating/electrical switching characteristics can be fabricated by synthesizing all the constituent nanotubes to be of the same diameter and with $\theta_{2}=120^{\circ}$.

a previous experiment ${ }^{15}$ where the simultaneous presence of an ac voltage on the source-drain channel and a dc voltage on the control/gate terminal resulted in an abrupt turn off, possibly due to defect mediated negative capacitance effects. $^{21}$

From the Y-CNT electrical characteristics it is seen that the mode of operation of the device is quite different from that of a conventional FET and more akin to a Schottkybarrier-type FET. ${ }^{22}$ The current modulation may be controlled by the electric field $\left(=\nabla_{\mathbf{r}} \cdot \mathbf{V}\right)$ at the gating terminal, and not the carrier density in the channel. In this case, the device performance can be improved by increasing the diameter of the gate so as to have a greater modulating effect on the source-drain current. The assumption of electric field control can also be tested ${ }^{23}$ by varying the angle $(\theta)$ between the branches of the Y-CNT. From elementary electrostatics, ${ }^{24}$ the surface-charge density at a distance $\rho$ from the intersection of the gate with the junction varies as $\rho^{(\pi / \theta-1)}$ which implies a greater modulating effect at a sharper angle. As an example, for the Y-CNT in Fig. 4(a), the stem would have a higher gating efficiency than in case (b). The manipulation of the junction region to be dielectric/insulating (say, by localized ion implantation) can also be used to change the electrochemical potential and vary device characteristics. It is interesting to note the similarity of Fig. 4(a) to a MOSFET, where the stem is analogous to a gate, the dielectric particle plays the role of the oxide, and the channel is formed by conduction through the branches. However, the latter option leads to a loss of flexibility in the Y-CNT. On the other hand, uniform electrical gating/switching characteristics could be obtained by making all the nanotubes the same diameter and length, at an angle of $120^{\circ}$ to each other [Fig. 4(b)].

We also suggest that even more interesting applications are facilitated by our experimental results and observations. For example, a single Y-CNT switching device can be made to have diverse operating characteristics through the use of different gating terminals. Y-junction based technology can be used for devices with (1) multiple programmable characteristics and as (2) components in field programmable gate arrays (FPGAs) for reconfigurable computing, ${ }^{25}$ where it would be possible to dynamically alter circuit paths. The large scale integration density and high speed of signal propagation intrinsic to nanotubes would be advantageous in this regard.

For such applications to be feasible, it would be important and necessary to gain better control of the geometry, through synthesis or processing, of Y-CNTs. It is plausible that remarkable features of Y-junction based transistors such as the abrupt switching ${ }^{15}$ and differential gain ${ }^{19}$ observed in earlier studies could be related to the presence and location of defects. In situ engineering of CNT morphology, e.g., exposure to intense electron-beam radiation ${ }^{13}$ could also be utilized to tailor individual Y-CNT characteristics.

The authors gratefully acknowledge support from the National Science Foundation (Grant No. ECS-05-08514) and the Office of Naval Research (Award No. N00014-06-10234). The authors also appreciate the use of facilities at the National Center for Electron Microscopy (NCEM) at the Lawrence Berkeley National Laboratory, Berkeley.

${ }^{1}$ P. Avouris, Acc. Chem. Res. 35, 1026 (2002).

${ }^{2}$ P. Avouris, J. Appenzeller, R. Martel, and S. Wind, Proc. IEEE 91, 1772 (2003).

${ }^{3}$ Z. Yao, H. W. C. Postma, L. Balents, and C. Dekker, Nature (London) 402, 273 (1999).

${ }^{4}$ P. G. Collins and P. Avouris, Sci. Am., 62 (2000).

${ }^{5}$ J. Hone, M. C. Llaguno, N. M. Nemes, A. T. Johnson, J. E. Fischer, D. A. Walters, M. J. Casavant, J. Schmidt, and R. A. Smalley, Appl. Phys. Lett. 77, 666 (2000).

${ }^{6}$ S. J. Tans, M. H. Devoret, H. Dai, A. Thess, R. E. Smalley, L. J. Geerligs, and C. Dekker, Nature (London) 386, 474 (1997).

${ }^{7}$ L. Forro and C. Schonenberger, in Carbon Nanotubes-Topics in Applied Physics, edited by P. Avouris (Springer, Heidelberg, 2001), Vol. 80, pp. 329-391.

${ }^{8}$ C. T. White and T. N. Todorov, Nature (London) 393, 240 (1998).

${ }^{9}$ J. Nygard, D. H. Cobden, M. Bockrath, P. L. McEuen, and P. E. Lindelof, Appl. Phys. A: Mater. Sci. Process. 69, 297 (1999).

${ }^{10}$ S. J. Tans, A. R. M. Verschueren, and C. Dekker, Nature (London) 393, 49 (1998).

${ }^{11}$ H. W. C. Postma, T. Teepen, Z. Yao, M. Grifoni, and C. Dekker, Science 293, 76 (2001).

${ }^{12}$ B. C. Satishkumar, P. J. Thomas, A. Govindaraj, and C. N. R. Rao, Appl. Phys. Lett. 77, 2530 (2000).

${ }^{13}$ M. Terrones, F. Banhart, N. Grobert, J.-C. Charlier, H. Terrones, and P. M. Ajayan, Phys. Rev. Lett. 89, 075505 (2002).

${ }^{14}$ C. Papadapoulos, A. Rakitin, J. Li, A. S. Vedeneev, and J. M. Xu, Phys. Rev. Lett. 85, 3476 (2000).

${ }^{15}$ P. R. Bandaru, C. Daraio, S. Jin, and A. M. Rao, Nat. Mater. 4, 663 (2005).

${ }^{16}$ N. Gothard, C. Daraio, J. Gaillard, R. Zidan, S. Jin, and A. M. Rao, Nano Lett. 4, 213 (2004).

${ }^{17}$ A. V. Melechko, V. I. Merkulov, T. E. McKnight, M. A. Guillorn, K. L. Klein, D. H. Lowndes, and M. L. Simpson, J. Appl. Phys. 97, 041301 (2005).

${ }^{18}$ V. Gopal, V. R. Radmilovic, C. Daraio, S. Jin, P. Yang, and E. A. Stach, Nano Lett. 4, 2059 (2004).

${ }^{19}$ B. R. Perkins, D. P. Wang, D. Soltman, A. J. Yin, J. M. Xu, and A. Zaslavsky, Appl. Phys. Lett. 87, 123504 (2005).

${ }^{20}$ H. Q. Xu, Appl. Phys. Lett. 80, 853 (2002).

${ }^{21}$ M. Beale and P. Mackay, Philos. Mag. B 65, 47 (1992).

${ }^{22}$ R. S. Muller and T. I. Kamins, Device Electronics for Integrated Circuits, 2nd ed. (Wiley, New York, 1986).

${ }^{23} \mathrm{P}$. Bandaru et al. (unpublished).

${ }^{24}$ J. D. Jackson, Classical Electrodynamics (Wiley, New York, 1999).

${ }^{25}$ J. Rabaey, Digital Integrated Circuits: A Design Perspective (Prentice Hall, New York, 1996). 